\title{
Pragmatists and Purists on CPT Invariance in Relativistic Quantum Field Theories
}

\author{
Jonathan Bain \\ Polytechnic Institute of New York University, 6 Metrotech Center, Brooklyn, NY 11201
}

Abstract. Philosophers of physics are split on whether foundational issues in relativistic quantum field theory (RQFT) should be framed within pragmatist approaches, which trade mathematical rigor for the ability to formulate non-trivial interacting models, or purist approaches, which trade the ability to formulate non-trivial interacting models for mathematical rigor. This essay addresses this debate by viewing it through the lens of the CPT theorem. I first consider two formulations of the CPT theorem, one purist and the other pragmatist, and extract from them a set of problems that clarifies the distinction between pragmatism and purity. I then apply this distinction to Greenberg's ([2002]) influential claim that the violation of CPT invariance in an interacting RQFT entails the violation of (restricted) Lorentz invariance. I show how this claim rests on an unsuccessful attempt to mediate between pragmatism and purity. I then evaluate another attempt at such mediation in the form of causal perturbation theory. This approach suggests that a focus on renormalized perturbation theory as a way of distinguishing pragmatists from purists may be misleading.

Keywords: quantum field theory, CPT theorem, causal perturbation theory

1. Introduction

2. Framing the Debate

3. Pragmatism versus Purity on CPT Invariance

3.1. The Axiomatic CPT Theorem

3.2. Weinberg's CPT Theorem

3.3. Pragmatism and Renormalization

4. Problems for Pragmatism and Purity

4.1. Three Problems for Pragmatism

4.2. Two Problems for Purity

4.3. The Product of Distributions

5. Greenberg on Relativity and CPT Invariance

6. Causal Perturbation Theory

5.1. A Purist Solution to the UV Problem

5.2. CPT Invariance

5.3. The Problem of Empirical Import and the Adiabatic Limit

7. Conclusion

Appendix

References

\section{Introduction}

Pragmatist approaches to relativistic quantum field theories (RQFTs) trade mathematical rigor for the ability to formulate non-trivial interacting models. Examples include the Lagrangian approach found in most textbooks (e.g., Kaku [1993]), and Weinberg's approach (e.g., Weinberg [1995]). Purist approaches to RQFTs trade the ability to formulate non-trivial interacting models for mathematical rigor. Examples include the axiomatic and algebraic formalisms (e.g., Streater and Wightman [2000]; Halvorson and Müger [2006]). Philosophers of physics are split on whether foundational issues related to RQFTs should be framed within pragmatist or purist approaches. Wallace ([2011]), for instance, has argued that "cutoff" quantum field theory (CQFT), a particular pragmatist approach, has been successful at resolving the mathematical and conceptual problems associated with renormalized perturbation theory, while axiomatic and algebraic quantum field theory (AQFT), which epitomize purist approaches, have failed; and this 
indicates that CQFT is the correct framework for philosophy of QFT. Fraser ([2011]), on the other hand, arguing in support of AQFT, has claimed that renormalization techniques illuminate the empirical structure of QFT, as opposed to its theoretical structure, and the success of these techniques is not the exclusive property of pragmatist approaches.

This essay addresses this debate by viewing it through the lens of a result that is generally considered to be unique to RQFTs; namely, the CPT theorem. This theorem entails that any state of a physical system described by an RQFT must possess CPT invariance; i.e., invariance under the combined transformations of charge conjugation $\mathrm{C}$, space inversion $\mathrm{P}$, and time reflection $\mathrm{T}$. In other words, the CPT theorem entails that CPT invariance is an essential property of RQFT states. Versions of this theorem appear in both pragmatist and purist approaches (see, e.g., Bain [2012]). While pragmatist versions of the theorem purport to include both free and interacting states, purist versions are restricted to free states at worst; or trivial, unrealistic interacting states at best. Greenberg ([2002]), however, has claimed that, within the purist axiomatic approach, a violation of CPT invariance in an interacting RQFT entails a violation of restricted Lorentz invariance. This claim makes no explicit provisos about whether the interacting states are trivial or not. It has been influential in both the physics and philosophy of physics literature, since it suggests a test for violations of Lorentz invariance via experiments that measure CPT violation (Kostelecky [2011]; Hagar [2009], p. 261). Moreover, in apparently linking Lorentz invariance with CPT invariance, it suggests the latter is somehow mysterious; in particular, some philosophers have wondered how the charge conjugation transformation $\mathrm{C}$ can arise from a purely spatiotemporal symmetry (Arntzenius [2011]; Greaves [2010]; Arntzenius and Greaves [2009]).

While questions like these may be well-motivated for other reasons, they cannot be underwritten by Greenberg's demonstration. Dütsch and Gracia-Bondía ([2012]) have argued that Greenberg's claim fails as an attempt to mediate between pragmatist and purist approaches. Their argument is framed within the context of 'causal perturbation theory', which seeks to establish a mathematically rigorous foundation for the perturbative techniques that underwrite pragmatist approaches (e.g., Gracia-Bondía [2006]). In fact, some authors have implicitly claimed that in causal perturbation theory one finds a reconciliation of pragmatism and purity (e.g., Helling [unpublished]). For instance, Brunetti and Fredenhagen ([2000], p. 624) observe that all analytic or algebraic approaches to RQFT '...by now seem to have missed the challenge for the concreteness needed by, say, particle physicists.' They go on to declare that '[a] notable exception is the rigorous formulation of perturbation theory [i.e., causal perturbation theory] which may be considered as interpolating between the world of phenomenological physics and the mathematical schemes mentioned above.'

The first part of this essay will frame Greenberg's claim, and Dütsch and Gracia-Bondía's critique of it, within the context of the debate between pragmatists and purists. The second part of the essay will consider the extent to which causal perturbation theory constitutes mediation between pragmatism and purity. On the one hand, causal perturbation theory is an axiomatic approach to RQFT, and faces the same problem that purist approaches face; namely, there are no non-trivial interacting models of its axioms. On the other hand, Dütsch and Gracia-Bondía ([2012]) provide a formulation of the CPT theorem within causal perturbation theory that, arguably, overcomes problems typically associated with purist formulations. Moreover, in its 
explicit adoption of perturbative techniques, causal perturbation theory also suggests that a focus on renormalized perturbation theory as a way of distinguishing pragmatists from purists may be misleading.

Section 2 sets the stage by framing the debate between Wallace ([2011]) and Fraser ([2011]) as one between pragmatism and purity. Section 3 then reviews and critiques two formulations of the CPT theorem, one purist and the other pragmatist. The problems extracted from these formulations are then summarized in Section 4, and applied in Section 5 to a critique of Greenberg's ([2002]) attempt to mediate between purity and pragmatism via CPT invariance. Finally, Section 6 evaluates causal perturbation theory with the intent of positioning it within the debate between pragmatists and purists.

\section{Framing the Debate}

The debate between pragmatists and purists centers on the question, 'What version of QFT should inform philosophical investigations of foundational issues?' A key aspect of this debate is what Wallace ([2011], p. 118) refers as the 'problem of renormalization':

As is widely known, the original attempts -- by Dirac, Jordan, Heisenberg and others -- to develop quantum field theory foundered on the problem of infinities: attempts to calculate physical quantities just led to divergent integrals. (Wallace [2011], p. 117.)

Wallace claims that the version of QFT he refers to as 'cutoff' or 'conventional' QFT (CQFT) solves this problem, whereas an alternative version, axiomatic or algebraic QFT (AQFT) does not. In CQFT, one inserts a finite energy cutoff $\Lambda$ into the theory and then uses it both as a means to demarcate the energy scale at which the theory is supposed to be valid, and as a means to regularize divergent integrals in perturbative expansions of the relevant quantities of interest. The cutoff serves to freeze out the short distance, high energy degrees of freedom of the theory, and one then adopts an agnostic attitude about what happens at energy scales above the cutoff. According to Wallace,

This, in essence, is how modern particle physics deals with the renormalization problem: it is taken to presage an ultimate failure of quantum field theory at some short lengthscale, and once the bare existence of that failure is appreciated, the whole of renormalization theory becomes unproblematic, and indeed predictively powerful in its own right. (Wallace [2011], p. 119.)

AQFT, on the other hand, addresses the problem of renormalization by attempting to identify a set of axioms that both free and interacting QFTs satisfy. But, '...no such theory has ever been found: the only known physically realistic algebraic quantum field theories in four dimensions are free-field theories' (Wallace [2011], p. 119). Wallace ([2011], p. 118) also suggests that CQFT and AQFT are 'rival research programs, trying in different ways to resolve the problem of renormalization'. CQFT assumes QFTs break down at short distances/high energies, while AQFT assumes QFTs can be defined on arbitrarily small spacetime regions (AQFTs define fields as operator-valued, or algebra element-valued, distributions). As rivals, Wallace believes there is a clear winner: 
CQFT makes a very large number of novel empirical predictions. Chief amongst these are the hundreds of cross-sections, decay rates, mass ratios, resonances, magnetic moments, and the like, calculable within the Standard Model and empirically confirmed: the only way we know to make mathematical and physical sense of the Standard Model is via CQFT, so it does not seem unreasonable to count the quantitative predictions of the Standard Model as predictions of CQFT. (If AQFT could also reproduce those predictions, they would equally count as novel predictions of AQFT; it can't.) (Wallace [2011], p. 119.)

Fraser ([2011]) seeks to defend AQFT against this critique. She first identifies the approach to QFT found in most textbooks as "Lagrangian" QFT (LQFT). This approach comes in two flavors: LQFT without cutoffs, and LQFT with cutoffs (Fraser [2011], p. 126). Wallace's CQFT is identified with 'LQFT with cutoffs in which renormalization is effected by the application of RG [viz., renormalization group] methods' (Fraser [2011], p. 132). The basis of Fraser's defense is a distinction between the theoretical content of QFT and its empirical content:

There is room for parallel research programs in QFT due to the fact that, in the context of QFT, there is a particularly distinct gap between the theoretical and empirical levels. The empirical evidence in support of QFT takes the form of the results of scattering experiments; these are encoded in the scattering matrix elements ( $S$-matrix elements). The theoretical content is, roughly speaking, the background apparatus that is used in the derivation of the $S$-matrix elements. (Fraser [2011], p. 127.)

Moreover, Fraser seeks to divorce the theoretical content of QFT from its empirical content:

The core theoretical content can also be explicated in the form of a set of theoretical principles. The theoretical principles of QFT are remote from the derived $S$-matrix elements; in LQFT the fact that renormalization is necessary is one manifestation of this. (Fraser [2011], p. 127.)

This applies in particular to Wallace's CQFT:

...RG methods illuminate the empirical structure of QFT. As such, a priori, the lessons drawn about the empirical structure of QFT are not the exclusive property of CQFT; it remains to be seen whether it is possible for constructive field theorists to come up with rigorous versions of the same techniques. (Fraser [2011], p. 132)

Fraser then argues that, while CQFT offers insight into the empirical structure of QFT, it does not offer a rigorous foundation for the theoretical content:

In my view, LQFT with cutoffs yields valuable insight into the empirical structure of QFT, but not the theoretical content" ([2011], p. 127).

In particular, by inserting a finite cutoff, CQFT sacrifices Lorentz invariance. Fraser also implies that, by adopting renormalization techniques (either naive or sophisticated), CQFT fails to provide a rigorously well-defined theoretical framework, whereas '...AQFT and its models are mathematically well-defined by design' (p. 130). And '[f]urthermore, the proofs of the CPT and 
spin-statistics theorems hinge on assumptions about the mathematical well-definedness of QFT' (p. 131).

The debate between Wallace and Fraser suggests that the role that renormalization plays in various versions of QFT is a fundamental issue, as is a separation between the theoretical content of an RQFT and its empirical content. In particular, both Wallace and Fraser suggest that renormalization is where QFTs make contact with empirical results, and Fraser in particular indicates that this aspect of a QFT can be separated from its theoretical content. Moreover, the procedure by means of which Wallace's CQFT makes novel empirical predictions involves not just renormalization, but in addition perturbation theory: It is the insertion of a cut-off into the integral terms that appear in perturbative power series expansions of relevant quantities that characterizes CQFT's freezing out of high-energy degrees of freedom. Thus one might identify 'renormalized perturbation theory' as what distinguishes the empirical content of Wallace's CQFT from the theoretical content of Fraser's AQFT.

This suggests the following general characteristics of the positions in the debate: Pragmatist approaches to QFT, like CQFT, directly engage with renormalized perturbation theory, and thus the empirical content of QFT. On the other hand, they face problems with mathematical rigor with respect to the theoretical content of the theory. Purist approaches to QFT, like AQFT, do not engage with renormalized perturbation theory, and so do not engage directly with the empirical content of the theory. On the other hand, they seek to place the theoretical content of QFT on rigorous mathematical foundations.

There seem to be two initial problems with this formulation of the debate. The first involves the distinction between the empirical content of an RQFT and its theoretical content. As noted above, Fraser suggests that the theoretical principles associated with a QFT are "remote" from derived quantities like the $S$-matrix; but there are formulations of QFT in which this is arguably not the case. For instance, Weinberg's ([1995]) approach makes an expression for the $S$-matrix, and various properties it is required to posses, central theoretical principles of the theory. In Weinberg's approach, it is arguably difficult to separate the empirical content of the theory from its theoretical content. More will be said of this approach in Section 3.2 below. Moreover, the problem of renormalization that Wallace identifies is, arguably, a bit more nuanced than either Wallace or Fraser indicate. There are two conceptually distinct types of infinities that beleaguer various formulations of QFTs. One involves divergences in the parameters used to define interacting states in terms of free states, and the other involves divergences in integrals that appear in perturbative power series expansions of relevant quantities. The first type may be associated with renormalized parameters, and thus a "problem of renormalization". The second type is typically associated with perturbation theory, and for some theories, the first type can be employed to effectively address the second type (such theories are "renormalizable"). However, this is generally not the case without further ado, and one can argue that the second type of infinity, ultimately, involves the definition of products of fields, whether free or interacting. These issues will be discussed in Sections 3 and 4 below.

Thus to fully appreciate the distinction between pragmatist and purist approaches to QFT, one should be aware that renormalization can be separated from perturbation theory, and that a lack 
of rigor with respect to one of these concepts need not entail a lack of rigor with respect to the other. To make this point a bit more concrete, in the following I will focus specifically on how two particular versions of pragmatism and purity formulate a result that is typically taken to be unique to RQFTs; namely, the CPT theorem.

\section{Pragmatism versus Purity on CPT Invariance}

This section critiques two formulations of the CPT theorem, one purist and the other pragmatist. The immediate goal is to identify explicit problems associated with each formulation that will make the distinction between pragmatism and purity more concrete.

\subsection{The Axiomatic CPT Theorem}

The first example of a formulation of the CPT theorem I'd like to consider is the purist axiomatic approach. In this approach, the basic objects are Wightman functions. These are first and foremost vacuum expectation values of unordered products of fields: $W^{(n)}\left(x_{1}, \ldots, x_{n}\right) \equiv$ $\left\langle 0\left|\phi\left(x_{1}\right) \ldots \phi\left(x_{n}\right)\right| 0\right\rangle$ for vacuum state $|0\rangle$ and field $\phi(x)$. Here $\phi(x)$ is intended to represent a generic quantum field of arbitrary spin, and $|0\rangle$ is the corresponding unique vacuum state. In early formulations of QFT, the fields were taken to be operator-valued functions; i.e., maps from spacetime to the space of linear operators acting on a Hilbert space of states. However, Bohr and Rosenfeld argued that a field at a point cannot define a linear operator (informally, the uncertainty relations entail a measurement at a point would yield infinite energy). ${ }^{1}$ The axiomatic approach addresses this issue by "smearing" the field in spacetime; i.e., defining it as a spacetime average, weighted by an arbitrary "test" function. More precisely, one defines a field as an operator-valued distribution,

$$
\phi(f)=\int_{-\infty}^{\infty} \phi(x) f(x) d^{4} x
$$

where $f$ is an arbitrary test function. This defines a field as a map from the space $\mathcal{D}\left(\mathbb{R}^{4}\right)$ of test functions to the space of linear operators on a Hilbert space. ${ }^{2}$ This map is required to be continuous; thus a distribution is an element of the dual space $\mathcal{D}^{\prime}\left(\mathbb{R}^{4}\right)$ consisting of continuous linear functionals on $\mathcal{D}\left(\mathbb{R}^{4}\right)$. An $n$-point Wightman function then defines a distribution $W^{(n)}\left(f_{1}, \ldots, f_{n}\right)=\left\langle 0\left|\phi\left(f_{1}\right) \ldots \phi\left(f_{n}\right)\right| 0\right\rangle$, which is an element of the space $\mathcal{D}^{\prime}\left(\mathbb{R}^{4 n}\right)$.

In addition to being vacuum expectation values of unordered products of fields, Wightman functions are required to satisfy a number of axioms, and it is the hope of advocates of this

\footnotetext{
${ }^{1}$ Halvorson and Müger ([2007], pp. 768-78) consider the extent to which such informal arguments can be made rigorous.

${ }^{2} \mathcal{D}\left(\mathbb{R}^{4}\right)$ is the space of continuous functions on $\mathbb{R}^{4}$ with compact support (i.e., continuous functions that vanish at infinity). For some applications, one uses the smaller Schwarz space $\mathcal{S}\left(\mathbb{R}^{4}\right)$ of continuous functions on $\mathbb{R}^{4}$ that decrease rapidly at infinity (such functions more readily admit Fourier transforms).
} 
approach that models of these axioms for interacting RQFTs can be constructed. ${ }^{3}$ For the purposes of deriving CPT invariance, the following three assumptions suffice.

(i) Restricted Lorentz invariance (RLI). The fields are required to be invariant under the restricted Lorentz group $L_{+}^{\uparrow}$, which is the subgroup of the Lorentz group connected to the identity that consists of Lorentz boosts but not parity or time reversal transformations.

(ii) Spectrum Condition (SC). The fields are required to possess positive energy, in the sense that the spectrum of the momentum operator associated with $L_{+}^{\uparrow}$ is required to be confined to the forward lightcone.

Assumptions (i) and (ii) entail that Wightman functions can be analytically extended to complexanalytic functions that are invariant under the (proper) complex Lorentz group $L_{+}(\mathbb{C})$ with unit determinant, which, importantly does contain parity and time reversal transformations. Moreover, the extended domain contains real points of analyticity referred to as "Jost points". 4 The third assumption is imposed with respect to these latter:

(iii) Weak Local Commutativity (WLC). At (or in the neighborhood of) a Jost point the fields are required to satisfy $\left\langle 0\left|\phi\left(f_{1}\right) \ldots \phi\left(f_{n}\right)\right| 0\right\rangle=i^{K}\left\langle 0\left|\phi\left(f_{n}\right) \ldots \phi\left(f_{1}\right)\right| 0\right\rangle$, where $K$ is the number of fermionic fields.

WLC requires the fields to satisfy local commutativity (i.e., the condition that they commute (bosonic) or anti-commute (fermionic) at spacelike separated distances) only when they appear in unordered products in vacuum expectation values. Jost ([1957]) showed that the conjunction of (i), (ii) and (iii) entails the existence an anti-unitary operator that combines the actions of C, P, and $\mathrm{T}$ transformations on fields, leaving them invariant. ${ }^{5}$ Schematically, the axiomatic CPT theorem then takes the following form (Streater and Wightman [2000], p. 150):

\section{$[(\mathrm{RLI}$ of fields) \& SC \& WLC] $\Rightarrow$ (CPT invariance of fields)}

There are two general problems with this axiomatic understanding of CPT invariance. First, it faces what might be called the Problem of Empirical Import: No non-trivial interacting models of the Wightman axioms exist, thus there are no such things as non-trivial interacting Wightman functions. This suggests that, under the axiomatic understanding of CPT invariance, the latter is restricted to free or trivially interacting RQFT states. This is unfortunate, since the evidence for CPT invariance in particular, and for the reliability of RQFTs in general, invariably comes from (non-trivial) interacting RQFTs.

\footnotetext{
${ }^{3}$ Expositions of the Wightman axioms can be found in (Streater and Wightman [2000]; Araki [1999]; Haag [1996]). In the following (in particular, Section 5), it will be important to distinguish a vacuum expectation value of unordered products of fields from a Wightman function. The latter are vacuum expectation values of unordered products of fields that, in addition, satisfy the Wightman axioms.

${ }^{4}$ A Jost point $\left(x_{1}, \ldots, x_{n}\right)$ is a convex set of points that are spacelike separated from each other. In other words, the difference variables $\xi_{i} \equiv x_{i-1}-x_{i}$ satisfy $\left(\sum_{j=1}^{n} \lambda_{j} \xi_{j}\right)^{2}<0$, for all $\lambda_{j} \geq 0$, with $\sum \lambda_{j}>0$ (see, e.g., Streater and Wightman [2000], p. 71; Greenberg [2006], p. 1545).

${ }^{5}$ Hermiticity of Wightman functions (one of the Wightman axioms) is also required for this existence proof.
} 
A second problem with the axiomatic understanding of CPT invariance is the fact that the product of two distributions at the same point is not in general well-defined. This might be called the UV Problem (Steinmann [2000], p. 73) since it is a problem with defining a quantity at short distances, or large energies. This makes the definition of a Wightman function as a product of operator-valued distributions problematic. A solution to this problem exists for free fields. Such fields can be decomposed into a linear sum of creation and annihilation operators $a(\mathbf{p})$, $a^{\dagger}(\mathbf{p})$ that act on multi-particle states in a Fock space. Products of such fields will thus consist of linear sums with terms involving combinations of $a(\mathbf{p})$ and $a^{\dagger}(\mathbf{p})$, and one can show that only combinations of the form $a^{\dagger}(\mathbf{p})^{n} a(\mathbf{p})^{m}$ correspond to well-defined Fock space operators (Reed and Simon [1975], pp. 219-20). Thus if one restricts one's attention to Wick polynomials; i.e., linear sums of products of fields in which all creation operators occur to the right of all annihilation operators, then such products are well-defined (Haag [1996], p. 45; Steinmann [2000], p. 51; Reed and Simon [1975], p. 225). However, this solution only works for fields that can be decomposed into linear sums of Fock space creation and annihilation operators, and for most non-trivial interacting fields, such a decomposition does not exist.

\subsection{Weinberg's CPT Theorem}

I'd now like to consider Weinberg's derivation of the CPT theorem as an example of a pragmatist approach. This approach takes the basic object of an RQFT to be the $S$-matrix, which encodes the probability amplitudes for particle scattering events (see, e.g., Weinberg [1964], [1995]). Weinberg ([1995]) bases this approach on three assumptions:

(i) Perturbation Theory. The $S$-matrix takes the form of a perturbative power series expansion in time-ordered products of an interaction Hamiltonian density $\mathscr{H}_{\text {int }}(x)$ :

$$
S_{\beta \alpha}=\sum_{n=0}^{\infty} \frac{-i^{n}}{n !} \int\left\langle\beta\left|T\left\{\mathscr{H}_{\text {int }}\left(x_{1}\right) \ldots \mathscr{H}_{\text {int }}\left(x_{n}\right)\right\}\right| \alpha\right\rangle d^{4} x_{1} \ldots d^{4} x_{n}
$$

where $|\beta\rangle,|\alpha\rangle$ are asymptotic multi-particle states, and the time-ordered product $T\left\{\mathscr{H}_{\text {int }}\left(x_{1}\right) \ldots \mathscr{H}_{\text {int }}\left(x_{n}\right)\right\}$ orders the $\mathscr{H}_{\text {int }}\left(x_{i}\right)$ according to $t_{1}>\ldots>t_{n}$.

(ii) Lorentz Invariance of the S-Matrix. The $S$-matrix is invariant under restricted-Lorentz transformations.

(iii) Cluster Decomposition of the S-matrix (CD). Let $S_{\beta_{1}+\ldots+\beta_{N}, \alpha_{1}+\ldots+\alpha_{N}}$ represent the $S$-matrix for $N$ multi-particle processes $\left|\alpha_{1}\right\rangle \rightarrow\left|\beta_{1}\right\rangle, \ldots,\left|\alpha_{N}\right\rangle \rightarrow\left|\beta_{N}\right\rangle$. If all particles in states $\left|\alpha_{i}\right\rangle,\left|\beta_{i}\right\rangle$ are spacelike separated from all particles in states $\left|\alpha_{j}\right\rangle,\left|\beta_{j}\right\rangle$, for $i \neq j$, then the $S$-matrix factorizes: $S_{\beta_{1}+\ldots+\beta_{N}, \alpha_{1}+\ldots+\alpha_{N}}=S_{\beta_{1} \alpha_{1}} \ldots S_{\beta_{N} \alpha_{N}}$.

$\mathrm{CD}$ is a locality constraint that imposes a factorizability condition on the $S$-matrix (Weinberg [1995], p. 177). It entails that scattering events in spacelike separated regions of spacetime do not interfere. Weinberg demonstrates that a sufficient condition ('the only known way', 
Weinberg [1964], p. 1318) for (iii) to be compatible with (ii) is that $\mathscr{H}_{\text {int }}(x)$ be a functional of fields that satisfy RLI and local commutativity (i.e., the fields commute (bosonic) or anticommute (fermionic) at spacelike separated distances), and that are linear combinations of Fock space creation and annihilation operators for free particles. Weinberg ([1995], pp. 198-9) then argues that if these fields carry a non-zero value of a conserved charge, then anti-particle states must be posited. CPT invariance of the full Hamiltonian density then follows from a consideration of how the relevant creation and annihilation operators transform under $\mathrm{C}, \mathrm{P}$, and $\mathrm{T}$ separately (see, e.g., Weinberg [1995], pp. 244-6). Thus in Weinberg's approach, the CPT theorem takes the following schematic form:

$[(\mathrm{RLI}$ of $S$-matrix) \& CD \& (existence of conserved charges) $] \Rightarrow(\mathrm{CPT}$ invariance of $\mathscr{H}(x))$

where $\mathscr{H}(x)$ is the full Hamiltonian density.

In Weinberg's approach, one may claim that CPT invariance is a property of both interacting and non-interacting states, in so far as the demonstration of CPT invariance of the full Hamiltonian density ultimately rests on CPT invariance of the creation and annihilation operators of free multi-particle states, that transform, under the $S$-matrix into interacting multi-particle states. However, the rigor of this claim is made problematic by the following considerations:

(a) First, the formula (2) for the $S$-matrix implicitly assumes that multi-particle states at asymptotic times are free, and can be unitarily related to interacting multi-particle states at finite times. This is made problematic by the consequences of Haag's theorem, which indicates that, under reasonable assumptions, the Hilbert spaces for interacting and free states belong to unitarily inequivalent representations of the canonical (anti-) commutation relations, thus a unitary $S$-matrix operator that transforms free states into interacting states does not exist (see, e.g., Earman and Fraser [2006]; Duncan [2012], pp. 359-70).

(b) Second, for the types of interacting QFTs of interest, many of the individual terms in the power series (2) diverge. This is a manifestation of the UV Problem in the axiomatic approach, to the extent that it involves defining the products of interacting fields at the same point that appear in (2).

(c) Finally, for the types of interacting QFTs of interest, it is not known if (2), considered as a formal power series, exists (i.e., is convergent). ${ }^{6}$ Call this the Existence Problem.

Problem (a) is implicitly addressed in pragmatist approaches by employing renormalization. Since philosophers have latched onto renormalization methods as a means to distinguish pragmatists from purists, it will help to see where in typical pragmatist approaches these methods are applied. This is the goal of the following section.

\footnotetext{
${ }^{6}$ This is certainly true for QED; however, there's some room for debate on whether theories that are asymptotically free, or asymptotically safe, face this problem (see, e.g., Boulatta and Butterfield [unpublished]).
} 


\subsection{Pragmatism and Renormalization}

In the following by a pragmatist approach, I will mean an approach that employs renormalized perturbation theory, as presented in typical textbooks on QFT. It will first be helpful to indicate how the formula (2) for the $S$-matrix is applied in such approaches. Formula (2) involves matrix elements of multi-particle states and time-ordered products of an interaction Hamiltonian density. Typical pragmatist approaches simplify (2) by reducing it to an expression that only involves vacuum expectation values of time-ordered products of fields, referred to hereafter as ' $\tau$ functions'. For instance, an $n$-point $\tau$-function is given by $\left\langle 0\left|T\left\{\phi\left(x_{1}\right), \ldots, \phi\left(x_{n}\right)\right\}\right| 0\right\rangle$, where $|0\rangle$ is the vacuum state and $\phi(x)$ a generic quantum field. One can distinguish between interacting $\tau$ functions and free $\tau$-functions, depending on whether the fields that appear in them are interacting or free (i.e., satisfy homogeneous or inhomogeneous field equations, respectively). The initial goal of pragmatist approaches is to reduce formula (2) to an expression that only involves free $\tau$-functions. ${ }^{7}$ In practice, this goal is achieved by appealing to the following two results: ${ }^{8}$

(i) One first makes use of a non-perturbative result due to (Lehmann, Symanzik and Zimmermann [1955]) that allows $S$-matrix elements to be calculated from interacting $\tau$ functions. This is known as the LSZ reduction formula and comes in many flavors, one per type of field. ${ }^{9}$ For instance, the LSZ reduction formula for a massive scalar field solution to the Klein-Gordon equation is given by:

$$
\begin{aligned}
{ }_{\text {out }}\left\langle\mathbf{p}_{1}, \ldots, \mathbf{p}_{n} \mid \mathbf{k}_{1}, \ldots, \mathbf{k}_{m}\right\rangle_{\text {in }} & =(i / \sqrt{Z})^{n+m} \int d^{4} x_{1} \ldots d^{4} y_{m} e^{-i p_{x} x_{i}+i k_{j} y_{j}} \prod_{i}\left(\partial_{x_{i}}^{2}+m^{2}\right) \prod_{j}\left(\partial_{y_{j}}^{2}+m^{2}\right) \\
& \times\left\langle 0\left|T\left\{\varphi\left(x_{1}\right) \ldots \varphi\left(x_{n}\right) \varphi\left(y_{1}\right) \ldots \varphi\left(y_{m}\right)\right\}\right| 0\right\rangle .
\end{aligned}
$$

The left-hand side of (3) represents an $S$-matrix element for $n$ incoming asymptotic particles with momenta $k_{i}$ and $m$ outgoing asymptotic particles with momenta $p_{i}$. The righthand side of (3) indicates how this $S$-matrix element can be calculated in terms of an interacting $\tau$-function, where $\varphi(x)$ is an interacting field (in this example, a solution to the inhomogeneous Klein-Gordon equation). The appearance of the constant $Z$ will be discussed immediately below.

(ii) One then assumes a perturbative split of the Hamiltonian $H=H_{0}+H_{\text {int }}$, into a nonperturbed piece $H_{0}$ (typically identified as the free Hamiltonian) and an interacting piece $H_{\text {int }}$ encoding (small) perturbations away from $H_{0}{ }^{10}$ The following formula due to (Gell-

\footnotetext{
${ }^{7}$ Once this goal has been achieved, standard pragmatist approaches then make use of Wick's theorem to reduce a free $n$-point $\tau$-function to a sum of products of free 2-point $\tau$-functions. The latter define Feynman propagators, and one can then employ the machinery of Feynman diagrams to calculate (2).

${ }^{8}$ These are discussed in (Bain [2000]). Textbooks vary on the order in which they present these results. Moreover, some textbooks forsake the second result by employing the path integral formalism to derive interacting $\tau$-functions as functional derivatives of a generating functional (see, e.g., Ryder [1996]). The order presented below follows (Maggiore [2005]) and (Kaku [1993]).

${ }^{9}$ A sample of textbook presentations of the LSZ formula includes (Duncan [2012], p. 286; Srednicki [2007], p. 51; Maggiore [2005], p. 115; Haag [1996], p. 83; Weinberg [1995], p. 437; Kaku [1993], p. 145).

${ }^{10}$ The Hamiltonian $H(t)$ is related to the Hamiltonian density $\mathscr{H}(x)$ by $H(t)=\int d^{3} \mathbf{x} \mathscr{H}(\mathbf{x}, t)$.
} 
Mann and Low [1951]) then allows interacting $\tau$-functions to be calculated from free $\tau$ functions:

$$
\left\langle 0\left|T\left\{\varphi\left(x_{1}\right) \ldots \varphi\left(x_{n}\right)\right\}\right| 0\right\rangle=\frac{\left\langle 0\left|T\left\{\phi_{I}\left(x_{1}\right) \ldots \phi_{I}\left(x_{n}\right) e^{-i \int H_{I} d t}\right\}\right| 0\right\rangle}{\left\langle 0\left|T\left\{e^{-i H_{I} d t}\right\}\right| 0\right\rangle}
$$

In this formula, the $\varphi\left(x_{i}\right)$ are interacting fields, the $\phi_{I}\left(x_{i}\right)$ are free fields in the interaction picture, and $H_{I}=e^{i H_{0}} H_{\text {int }} e^{-i H_{0}}$ is the interaction picture representation of $H_{\text {int }}{ }^{11}$ The lefthand side of (4) represents an interacting $\tau$-function that enters into the LSZ formula, and the right-hand side indicates how it can be calculated in terms of free $\tau$-functions.

In the LSZ formula (3), the constant $Z$ is a renormalization constant. The purpose of this constant is to relate the interacting field $\varphi(x)$ to free fields $\phi_{\text {in }}(x), \phi_{\text {out }}(x)$ at asymptotic times. In particular, one assumes,

$$
\langle\beta|\varphi(x)| \alpha\rangle \underset{t \rightarrow-\infty}{\longrightarrow} \sqrt{Z}\left\langle\beta\left|\phi_{\text {in }}(x)\right| \alpha\right\rangle, \quad\langle\beta|\varphi(x)| \alpha\rangle \underset{t \rightarrow+\infty}{\longrightarrow} \sqrt{Z}\left\langle\beta\left|\phi_{\text {out }}(x)\right| \alpha\right\rangle
$$

where $|\beta\rangle,|\alpha\rangle$ are free multi-particle states. Under this weak notion of convergence, matrix elements of interacting fields are required to converge to matrix elements of free, asymptotic fields at asymptotic times (as opposed to requiring that interacting fields converge directly to free fields). One way to motivate this assumption is by considering the action of a free asymptotic field on the vacuum with respect to a single-particle state (see, e.g., Duncan [2012], p. 282; Srednicki [2007], p. 38). An asymptotic single-particle 'in' state $|\mathbf{k}\rangle$ satisfies $\left\langle\mathbf{k}\left|\phi_{\text {in }}(x)\right| 0\right\rangle=$ $\left\langle\mathbf{k}\left|\phi_{\text {in }}(0)\right| 0\right\rangle=1$. Since an interacting field does not have a simple decomposition in terms of creation and annihilation operators, one would not expect $\langle\mathbf{k}|\varphi(0)| 0\rangle$ to equal 1; in particular, one may set it equal to $\sqrt{Z}$, for some constant $Z$. The assumption (5) may be considered a generalization of this. ${ }^{12}$ Formally, the renormalization constant $Z$ can be explicitly removed from the LSZ formula by replacing the "bare" interacting field with a renormalized interacting field defined by $\varphi_{r}(x) \equiv Z^{-1 / 2} \varphi(x)$. This assignment then guarantees that the renormalized interacting field behaves like the free field with respect to single-particle states; namely, $\left\langle\mathbf{k}\left|\varphi_{r}(0)\right| 0\right\rangle=1$.

Renormalization also explicitly enters into the derivation of the Gell-Man/Low formula (4). In particular, (4) assumes that the vacuum state is the ground state of the non-perturbed Hamiltonian $H_{0}$, and that the full Hamiltonian $H$ does not polarize the vacuum; in other words, one assumes $H_{0}|0\rangle=0$ and $H|0\rangle=0$. Since the full Hamiltonian is a functional of interacting

\footnotetext{
${ }^{11} \mathrm{~A}$ free field in the interaction picture is defined by $\phi_{I}(\mathbf{x}, t)=e^{i H_{0}\left(t-t_{0}\right)} \phi\left(\mathbf{x}, t_{0}\right) e^{-i H_{0}\left(t-t_{0}\right)}$, where $\phi\left(\mathbf{x}, t_{0}\right)$ is a free field at time $t_{0}$. A sample of textbook presentations of the Gell-Mann/Low formula includes (Duncan [2012], p. 246; Maggiore [2005], p. 119; Haag [1996], p. 65; Weinberg [1993], p. 288; Kaku [1993], p. 151).

${ }^{12}$ To derive the LSZ formula (3), one first obtains an expression for an asymptotic single-particle state, say $|\mathbf{k}\rangle_{\text {in }}$, in terms of an asymptotic free field acting on the vacuum: $|\mathbf{k}\rangle_{\text {in }}=a_{i n}^{\dagger}(\mathbf{k})|0\rangle=-i\left(E_{\mathbf{k}}\right)^{1 / 2} \int d^{3} \mathbf{x} e^{-i k x} \stackrel{\leftrightarrow}{\partial}_{0} \phi_{i n}(x)|0\rangle$ (where the last equality follows by inverting the expression for $\phi_{i n}(x)$ as a linear sum of creation and annihilation operators). The LSZ formula is obtained by applying this expression and (5) to an $S$-matrix element to systematically replace single-particle asymptotic states with expressions involving interacting fields.
} 
fields which do not, in general, admit a decomposition into creation and annihilation operators, the latter condition is typically not guaranteed. To enforce it, one defines a renormalized Hamiltonian $H_{r}=H-\Delta$. This corresponds to renormalizing the mass that appears in the interacting theory. If the latter is given by $m_{B}$ (the "bare" mass), and the shift corresponding to $\Delta$ is given by $\delta m$, then the renormalized mass $m_{r}$ (the "physical" mass) is given by $m_{r}{ }^{2} \equiv m_{B}{ }^{2}+$ $\delta m^{2}$.

Thus, from a conceptual standpoint, renormalization is imposed to force the behavior of the interacting theory to conform to the behavior of the free theory, as far as the ground state and single-particle states are concerned. ${ }^{13}$ At this point, two things should be made clear: The first is how renormalization addresses problem (a) in Section 3.2 above; and the second is the extent to which renormalization is independent of perturbation theory; i.e., the extent to which problem (a) is independent of the $U V$ and Existence Problems (problems (b) and (c)). Renormalization solves problem (a) in the following sense: The renormalized field and the renormalized Hamiltonian are not self-adjoint operators (formally, for typical interacting theories, the renormalization constant $Z$ and the mass shift $\delta m$ are infinite). This entails, for instance, that $H_{r}$ does not implement unitary time translations, contrary to one of the assumptions of Haag's theorem (Fraser [2009], p. 547). Whether this constitutes an adequate solution to problem (a) will depend on your mathematical proclivities. The fact that renormalized parameters are formally infinite will upset mathematical purists. For such purists, the renormalization procedure simply replaces problem (a) with another problem, call it the Renormalization problem for pragmatists.

That renormalization is independent of perturbation theory is already indicated above, insofar as renormalization appears already in the non-perturbative derivation of the LSZ formula (as well as in the non-perturbative Källen-Lehman representation of the interacting Feynman propagator mentioned in footnote 12). Perturbation theory enters explicitly into the derivation of the GellMann/Low formula, which in practice is calculated by expanding the exponentials on the righthand side of (4) as power series in the coupling constants that appear in $H_{I}$. Problems (b) and (c) then re-emerge as problems associated with these power series.

Thus in principle, renormalization is independent of perturbation theory, and hence problem (a) is, in principle, independent of problems (b) and (c) in Section 3.2 above. As Weinberg states:

...the renormalization of masses and fields has nothing directly to do with the presence of infinities, and would be necessary even in a theory in which all momentum space integrals were convergent (Weinberg [1995], p. 441).

Similar remarks are also made by Steinmann:

Concerning the relation between renormalization and the removal of UV divergences it must be stressed that these are at first hand quite different problems. Renormalization... is necessary

\footnotetext{
${ }^{13}$ Another way to see this is by an analysis of the non-perturbative Källen-Lehman representation of the interacting 2-point $\tau$-function (Weinberg [1995], pp. 439-49).
} 
independent of the occurrence of UV divergences, if we want to describe the theory in terms of directly measureable parameters... (Steinmann [2000], p. 83).

However, there is a formal sense in which renormalization solves the UV Problem (problem (b)). The types of divergences associated with perturbative expansions of interacting $\tau$-functions can in principle be regularized by either renormalizing the parameters that are present in the Lagrangian density, or by introducing new renormalized parameters. For example, in ' $\varphi^{4}$ theory (i.e., massive scalar field theory with a quartic interaction term $\lambda \varphi^{4}$ ), the divergences that appear in the perturbative expansion of the 2-point $\tau$-function can be regulated by renormalizing the mass and the field, whereas the divergences that appear in the perturbative expansion of the 4point $\tau$-function can be regulated by renormalizing the coupling constant $\lambda .{ }^{14}$ In principle, all divergences in higher-order $\tau$-functions can also be regularized by introducing new terms into the Lagrangian density and renormalizing the parameters associated with them. The 6-point $\tau$ function, for instance, can be regularized by introducing a new term $\lambda_{(6)} \varphi^{6}$ into the Lagrangian density and renormalizing the parameter $\lambda_{(6)}$. In general, if this process never ends; i.e., if for each higher-order $\tau$-function one needs to introduce new terms in the Lagrangian density in order to regularize it, then the theory is referred to as non-renormalizable. On the other hand, if only a finite number of renormalized parameters are needed to regularize all possible divergent terms in the perturbative expansions of $\tau$-functions, then the theory is referred to as renormalizable. Thus, formally, renormalization solves Problem (b), for both non-renormalizable and renormalizable theories. However, since the solution to the problem for non-renormalizable theories requires an infinite number of renormalized parameters, and since the values of these parameters are fixed by experiments, a non-renormalizable theory has no predictive power, all things being equal. Thus, without further ado, renormalization only solves Problem (b) for renormalizable theories. ${ }^{15}$

\section{Problems for Pragmatism and Purity}

The problems identified Section 3 are not unique to the Wightman axiomatic approach and Weinberg's approach, nor are they restricted to the particular context of the CPT theorem. Rather, I'd like to suggest that they characterize purity and pragmatism, in general. This section briefly summarizes these problems.

\subsection{Three Problems for Pragmatism}

Pragmatists may be characterized as endeavoring to bring RQFTs into contact with experiment by employing renormalized perturbation theory. As indicated in Sections 3.2 and 3.3 above, this endeavor generates the following problems:

\footnotetext{
${ }^{14}$ For this description of renormalization in $\varphi^{4}$ theory, see (Maggiorie [2005], p. 135).

${ }^{15}$ One can make further ado by inserting a cut-off, as in Wallace's CQFT, and then demonstrating that, within the energy range specified by the cut-off, only a finite number of 'relevant' terms need to be renormalized in order to derive predictions from the theory. This further ado forms the basis of the effective field theory approach to QFTs.
} 
(1) The Renormalization Problem. This is the problem of employing infinitely renormalized parameters to relate interacting fields and free asymptotic fields in order to guarantee a non-trivial $S$-matrix.

(2) The UV Problem. This is the problem of the existence of divergent terms at high energies/short distances in the perturbative power series expansion of relevant quantities (i.e., the $S$-matrix, and/or $\tau$-functions). ${ }^{16}$

(3) The Existence Problem. This is the problem of whether or not the perturbative power series expansions of relevant quantities exist as convergent series.

These problems are not necessarily independent of each other, but they are, arguably, distinct. As noted in Section 3.3, while renormalization solves the UV Problem (in principle for both renormalizable and non-renormalizable theories), it is a conceptually distinct issue, and the problem it generates (i.e., the formal appearance of infinities in renormalized parameters) is conceptually distinct from the infinities associated with the $U V$ Problem. Moreover, the $U V$ Problem is also conceptually distinct from perturbation theory. The divergent terms in perturbative expansions of relevant quantities in interacting QFTs ultimately involve products of quantum fields at the same point; and just as it was early on determined that a quantum field at a point does not correspond to a well-defined operator on a Hilbert space, neither do products of quantum fields at the same point. As we'll see below, this problem carries over into purist approaches to QFT. ${ }^{17}$

\subsection{Two Problems for Purity}

Purity was originally motivated by the desire to rigorously address the pragmatist problems above. In particular, the Renormalization and Existence problems are addressed in (standard) axiomatic and algebraic approaches by rejecting renormalized perturbation theory and attempting to construct interacting models from a fundamental set of axioms applicable to both free and interacting theories. The considerations in Section 3.1 suggest that purists face the following two problems:

(1') The Problem of Empirical Import. This is the problem of making contact with experiment; i.e., the problem of constructing non-trivial interacting models of purist axioms.

\footnotetext{
${ }^{16}$ There is also an IR (infrared) version of this problem, which concerns the presence of divergent terms at low energies/long distances in perturbative expansions of relevant quantities. This IR Problem is primarily of concern for theories with massless interactions, like quantum electrodynamics.

${ }^{17}$ In addition to the three problems above, Steinman ([2000], p. 73) identifies the problem of how to determine the subset of solutions to the field equations of a QFT that should be deemed physically relevant. Call this the Physical Relevancy Problem. The standard way of addressing this problem requires that physically relevant solutions satisfy equal-time canonical (anti-) commutation relations (CCR). This is motivated by the similar requirement in quantum mechanics for systems with finite degrees of freedom. In the field-theoretic case, however, the systems have infinite degrees of freedom, and Stone's theorem then entails that unitarily inequivalent representations of the CCR will exist. This then generates a Representation Problem; namely, a problem of selecting which representation of the CCR to adopt. This Representation Problem is a key motivation for some advocates of the algebraic approach who seek physical relevancy at the level of the structure of the algebra of the CCR, as opposed to any concrete Hilbert space representation of it.
} 
(2') The UV Problem. This is the same problem that afflicts pragmatist approaches, and manifests itself for purists as the general problem of defining the product of distributions at the same point.

The basic criticism of purity is that, in its rejection of renormalized perturbative techniques, it rejects that aspect of pragmatist approaches that makes contact with experiment. The $U V$ Problem, as indicated above, is not a consequence of adopting perturbative techniques; rather, it is a problem associated with defining products of fields, and thus in principle can make trouble for purist approaches. Recall that a field at a point does not define a linear operator. The purist solution is to define a field as a distribution (operator-valued in the case of the Wightman axiomatic approach, algebra-element-valued in the case of the algebraic approach). A product of fields at the same point also does not define a linear operator, but simply replacing this product with a product of distributions does not necessarily produce an operator, essentially because a product of distributions at the same point is not always well-defined.

\subsection{Products of Distributions}

At this point, it may be worthwhile to consider the sense in which the product of distributions at the same point is problematic. While point-wise multiplication of functions is well-defined, this is not always the case for distributions. A distribution, as a continuous linear functional on a space of test functions, is supposed to be a 'generalized function'; thus how one defines the product of distributions will depend, in part, on the space of test functions one adopts, as well as the type of functions one desires to generalize. Consider the case of locally integrable functions (see, e.g., Kanwal [2004], p. 18, 22). A function $f(x)$ on $\mathbb{R}^{n}$ is locally integrable just when $\int_{A}|f(x)| d^{n} x$ exists for every bounded region $A$ in $\mathbb{R}^{n}$. Every locally integrable function $f(x)$ defines a regular distribution via $f(g)=\int f(x) g(x) d^{n} x$, for any test function $g \in \mathcal{D}$. Now consider the functions $f(x)=g(x)=1 / x^{1 / 2}$ (after Kanwal [2004], p. 32). Both $f(x)$ and $g(x)$ are locally integrable and thus each defines a regular distribution. However their product $f(x) g(x)=$ $1 / x$ is not locally integrable (since it fails to be integrable on any neighborhood of the origin), and thus does not define a regular distribution. Note that the integral $\int g(x) x^{-1} d^{n} x$ is well-defined on test functions $g$ restricted to the complement of the origin $\mathbb{R}^{n} \backslash\{0\}$.

Of more interest in QFT are products of Heaviside functions $\theta\left(t-t^{\prime}\right)$ and vacuum expectation values of unordered products of fields $\left\langle 0\left|\phi\left(x_{1}\right) \ldots \phi\left(x_{n}\right)\right| 0\right\rangle .{ }^{18}$ The Heaviside function $\theta\left(t-t^{\prime}\right)$ is 1 if $t-t^{\prime}>0$, and vanishes otherwise; thus an expression like

$$
\theta\left(t_{1}-t_{2}\right) \ldots \theta\left(t_{n-1}-t_{\mathrm{n}}\right)\left\langle 0\left|\phi\left(x_{1}\right) \ldots \phi\left(x_{n}\right)\right| 0\right\rangle
$$

is non-zero just when the product $\phi\left(x_{1}\right) \ldots \phi\left(x_{n}\right)$ is time-ordered according to $t_{1}>\ldots>t_{n}$. The $\tau$ functions of Section 3.3 that play essential roles in pragmatist's formulations of interacting RQFTs are linear sums of products of the form (6). Recall the basic strategy of pragmatist approaches: The LSZ formula (3) reduces $S$-matrix elements to expressions involving

\footnotetext{
${ }^{18}$ Recall from Section 3.1 that if these latter satisfy the Wightman axioms, they are referred to as Wightman functions.
} 
interacting $\tau$-functions, the Gell-Mann/Low formula (4) then expresses interacting $\tau$-functions in terms of free $\tau$-functions, and Wick's theorem then expresses any free $n$-point $\tau$-function as a sum of products of free 2-point $\tau$-functions. It is products of these latter, which define Feynman propagators $\Delta_{F}$, which are ultimately of interest in pragmatist descriptions of interacting RQFTs. A generic Feynman propagator is given by $\Delta_{F}\left(x, x^{\prime}\right)=\theta\left(t-t^{\prime}\right)\left\langle 0\left|\phi(x) \phi\left(x^{\prime}\right)\right| 0\right\rangle+$ $\theta\left(t^{\prime}-t\right)\left\langle 0\left|\phi\left(x^{\prime}\right) \phi(x)\right| 0\right\rangle$. As in the example in the previous paragraph, both $\theta\left(t-t^{\prime}\right)$ and $\left\langle 0\left|\phi(x) \phi\left(x^{\prime}\right)\right| 0\right\rangle$ define regular distributions. ${ }^{19}$ The questions then are, does their product define a distribution, too; and, in general, do products of Feynman propagators define distributions?

Reed and Simon ([1975], p. 90) address these questions with the following considerations (see, also, Gracia-Bondía [2006], p. 29). First, one notes that, when it exists, the Fourier transform $F[f g]$ of the product $f g$ of functions $f, g$ is given by the convolution of the Fourier transforms of $f$ and $g$; in other words, $F[f g]=(2 \pi)^{-n / 2} F[f] * F[g]$, where the convolution operator $*$ is given by $f_{1} * f_{2}(p) \equiv \int f_{1}(k) f_{2}(p-k) d^{n} k$. This motivates the following definition:

Def. 1. Let $t, u \in \mathcal{D}^{\prime}\left(\mathbb{R}^{n}\right)$. Then $w \in \mathcal{D}^{\prime}$ is the product of $t$ and $u$ if and only if for each $x \in$ $\mathbb{R}^{n}$, there exists some function $\varphi \in \mathcal{D}$, with $\varphi=1$ near $x$ so that for all $p \in \mathbb{R}^{n}$.

$$
F\left[\varphi^{2} w\right](p)=(2 \pi)^{-n / 2} \int F[\varphi t](k) F[\varphi u](p-k) d^{n} k
$$

The definition says that the product of two distributions is the inverse Fourier transform of the convolution of their Fourier transforms. ${ }^{20}$ One can now show that the Feynman propagator $\Delta_{F}$ satisfies Def. 1 (Gracia-Bondía [2006], p. 31; Reed and Simon [1975], p. 108). However $\left(\Delta_{F}\right)^{2}$ and higher powers of the Feynman propagator do not (Gracia-Bondía 2006, p. 31; Brunetti and Fredenhagen [2000], p. 632). In particular, one can show that for $n \geq 2,\left(\Delta_{F}\right)^{n}$ does not satisfy Def. 1 on the diagonal set $D_{n}$ of points in $\mathbb{R}^{4 n}$ defined by $D_{n}=\left\{\left(x_{1}, \ldots, x_{n}\right) \in \mathbb{R}^{4 n} \mid x_{1}=x_{2}=\ldots x_{n}\right\}^{21}$ In other words, such products are not defined at the same point. Translational invariance entails that $D_{n}$ is equivalent to the origin; thus one concludes that, for $n \geq 2,\left(\Delta_{F}\right)^{n}$ only satisfies Def. 1 on the complement of the origin $\mathbb{R}^{4 n} \backslash\{0\}$.

The upshot of this brief excursion into distribution theory is the following: The distributions of interest in interacting RQFTs contain products of interacting fields at the same point, and (under the above definition of the product of distributions), such products do not exist; rather, products of interacting fields are only defined on the complement of the origin in configuration space. In the next section, we'll see how this impacts an influential claim about the relation between CPT invariance and Lorentz invariance in interacting RQFTs.

\footnotetext{
${ }^{19}$ The Heaviside function defines a regular distribution by $\theta(f)=\int_{\mathrm{x}_{0} \geq 0} f(x) d^{4} x$. The form of the 2-point function $\left\langle 0\left|\phi(x) \phi\left(x^{\prime}\right)\right| 0\right\rangle$ depends on the nature of $\phi(x)$. For a neutral scalar field, $\left\langle 0\left|\phi(x) \phi\left(x^{\prime}\right)\right| 0\right\rangle=(2 \pi)^{-3} \int\left(2 E_{k}\right)^{-1} e^{-k\left(x-x^{\prime}\right)} d^{3} k$.

${ }^{20}$ In this definition, the function $\varphi$ serves to localize the distributions $t, u$; this guarantees that their Fourier transforms exist. Such localization is required for test functions in $\mathcal{D}$, but not $\mathcal{S}$ (see footnote 2).

${ }^{21}$ The details involve formulating a sufficient condition for Def. 1 by means of wave front analysis, and then demonstrating that a product of Feynman propagators fails to satisfy this criterion on $D_{n}$.
} 


\section{Greenberg on Relativity and CPT Invariance}

Greenberg ([2002]) claims that if CPT invariance is violated in an interacting QFT, then so is Lorentz invariance. His argument should be of interest to philosophers of physics, not only for its link between Lorentz invariance and CPT invariance, but also because it is presented in the axiomatic approach to QFT. As noted in Section 2, the axiomatic CPT theorem should be viewed as restricting CPT invariance to free (or trivially interacting) states, whereas pragmatist approaches to the CPT theorem (like Weinberg's) may be taken to predicate CPT invariance on both free and (non-trivially) interacting states (on pain of confronting the Renormalization Problem). This suggests that Greenberg's approach to CPT invariance is an attempt to reconcile purity with pragmatism. As we'll see below, this attempt fails.

Greenberg's argument begins by considering the following expression for an $n$-point $\tau$-function:

$$
\tau^{(n)}\left(x_{1}, \ldots, x_{n}\right) \equiv \sum_{p} \theta\left(t_{p_{1}}-t_{p_{2}}\right) \ldots \theta\left(t_{p_{n-1}}-t_{p_{n}}\right) W^{(n)}\left(x_{p_{1}}, \ldots, x_{p_{n}}\right)
$$

where the product of Heaviside functions $\theta\left(t_{p_{1}}-t_{p_{2}}\right) \ldots \theta\left(t_{p_{n-1}}-t_{p_{n}}\right)$ enforces the time ordering $t_{p_{1}}>\cdots>t_{p_{n}}$ on the Wightman function $W^{(n)}$, and the sum is over all permutations of the indices. As Section 3.3 indicated, the significance of $\tau$-functions is that $S$-matrix elements in pragmatist approaches can be reduced to expressions involving interacting $\tau$-functions (via the LSZ formula), and these latter can be expressed in terms of free $\tau$-functions (via the Gell-Mann/Low formula). However, in Section 4.3, $\tau$-functions were characterized as linear sums of products of Heaviside functions and vacuum expectation values of unordered products of fields $\left\langle 0\left|\phi\left(x_{1}\right) \ldots \phi\left(x_{n}\right)\right| 0\right\rangle$. Greenberg's starting point in (7) is the assumption that these latter satisfy the Wightman axioms, and thus can be replaced with Wightman functions $W^{(n)}\left(x_{1}, \ldots, x_{n}\right)$. It is this assumption that characterizes Greenberg's attempt to bridge the gap between pragmatism and purity.

Given (7), Greenberg demonstrates that, at a Jost point, restricted Lorentz invariance of $\tau^{(n)}$ entails that $W^{(n)}$ satisfies weak local commutativity (WLC). ${ }^{22}$ Thus if we require Wightman functions to satisfy RLI and SC, then a violation of CPT invariance of Wightman functions entails a violation of RLI of $\tau$-functions, as defined by (7). Schematically,

$$
\begin{aligned}
\left\{\left(\mathrm{RLI} \text { of } \tau^{(n)} \text { at Jost points) \& }(7)\right\}\right. & \left.\Rightarrow \text { (WLC of } W^{(n)} \text { at Jost points }\right) \\
& \Rightarrow \text { (CPT invariance of } W^{(n)} \text { that satisfy RLI and SC) }
\end{aligned}
$$

where the second entailment follows from Jost's proof of CPT invariance for Wightman functions (see Section 3.1). Greenberg concludes that if Lorentz invariance of an interacting RQFT requires restricted Lorentz invariance of $\tau$-functions, then '...if CPT invariance is violated in an interacting quantum field theory, then that theory also violates Lorentz invariance' ([2002], p. 1). ${ }^{23}$

\footnotetext{
${ }^{22}$ See Section 3.1 for the definition of WLC, and footnote 4 for the definition of a Jost point. An attempt to formalize Greenberg's informal argument is given in the Appendix.

${ }^{23}$ Greenberg ([2002], p. 1) defines a quantum field theory to be 'Lorentz covariant in cone' just when 'vacuum matrix elements of unordered products of fields (Wightman functions) are covariant'. This amounts to the
} 
This demonstration has been very influential in the physics literature, since it suggests a test for violations of Lorentz invariance via experiments that measure CPT violation (Gracia-Bondía, [private communication]; Kostelecky [2011]). Moreover, it appears to have influenced some recent accounts of CPT invariance in the philosophical literature. In a discussion of deviations from Lorentz invariance in approaches to quantum gravity, Hagar ([2009], p. 261) observes that '[i]t was also admitted that the predicted deviation was mediated through the breaking of CPT symmetry', and that '...the CPT theorem... says that violations of CPT symmetry imply violations of Lorentz invariance, but not vice versa.' Moreover, in an article on a geometrical understanding of the CPT theorem, Greaves ([2010], p. 28) poses the question 'How can it come about that one symmetry (e.g., Lorentz invariance) entails another (e.g., CPT) at all?' Greaves ([2010], p. 28) also poses the question 'How can there be such an intimate relationship between spatiotemporal symmetries (Lorentz invariance, parity reversal, time reversal) on the one hand, and charge conjugation, not obviously a spatiotemporal notion at all, on the other?' This is echoed in (Arntzenius [2011], p. 634), and (Arntzensius and Greaves [2009], p. 584). ${ }^{24}$

As a proof within the framework of the axiomatic approach, however, Greenberg's demonstration faces two difficulties.

(a) Since non-trivial interacting Wightman functions do not exist, neither do non-trivial interacting $\tau$-functions on Greenberg's definition (7) of the latter. More specifically, Jost's ([1957]) proof of CPT invariance assumes that Wightman functions satisfy RLI, SC, and WLC. The Spectrum Condition, SC, is important in order to establish that the complex extensions of the Wightman functions are analytic, and '...for non-trivial realistic models one cannot ascertain analyticity of Wightman-like functions' (Dütsch and Gracia-Bondía [2012], p. 429).

(b) The product of Heaviside functions and Wightman functions in (7) is not well-defined. As noted in Section 4.3 above, for $n \geq 2$, an $n$-point $\tau$-function, as a linear sum of products of Heaviside functions and vacuum expectation values of unordered products of fields, only exists as a distribution on the complement of the origin; i.e., $\tau^{(n)} \in \mathcal{D}^{\prime}\left(\mathbb{R}^{4 n} \backslash\{0\}\right)$. Since Greenberg's $\tau$-functions (7) are just $\tau$-functions in the sense of Section 4.3 that, in addition, have the property that the vacuum expectation values of unordered products of fields satisfy the Wightman axioms, they, too, are only defined on the complement of the origin.

assumptions of RLI and SC for Wightman functions, with the condition of being covariant in cone corresponding to SC (Greenberg [2002], p. 4, footnote 1). He defines a quantum field theory to be '[Lorentz] covariant out of cone' just when 'vacuum matrix elements of time-ordered products ( $\tau$ functions) are covariant'. This amounts to upholding RLI for $\tau$-functions, but denying SC. He then considers a QFT to be Lorentz invariant if it is Lorentz covariant both in and out of cone.

${ }^{24}$ The answer to this last question doesn't appear particularly mysterious, if one notes that restricted Lorentz invariance is not the only assumption needed to derive CPT invariance. For instance, in the axiomatic approach, the Spectrum Condition (SC) arguably explains why PT must be accompanied by C (Araki [1999], p. 158; Greenberg [2006], pp. 1547-8). Briefly, consider the generator of finite time translations, exp it $P^{0}$. If one desires to reverse the direction of time translations and simultaneously keep the energy $P^{0}$ positive (i.e., uphold SC), one needs to effect the transformation $i \rightarrow-i$, and charge conjugation secures this. In other words, the PT transformation must be antiunitary in order to uphold SC. (This is acknowledged by Greaves and Thomas [preprint], pg. 8.) 
Claim (a) is the purist's Problem of Empirical Import from Section 4, while claim (b) is the purist's UV Problem. Thus Greenberg's claim cannot be considered a purist claim about CPT invariance in a (non-trivial) interacting RQFT. Moreover, it's not entirely clear what significance his claim has as a purist claim about CPT invariance in a free (or trivially interacting) purist RQFT. What Greenberg shows is that a violation of CPT invariance in free (or trivially interacting) Wightman functions entails a violation of RLI in free (or trivially interacting) $\tau$-functions as defined by (7). But without further ado, and in the absence of the pragmatist's LSZ and Gell-Mann/Low formulas, the significance of free (or trivially interacting) $\tau$-functions in a purist RQFT is unclear.

Does Greenberg's claim fair any better under a pragmatist's understanding of CPT invariance? Pragmatists can accept the existence of interacting $\tau$-functions (on pain of confronting the Renormalization Problem), either via the Gell-Mann/Low formula (4), or its path integral equivalent. They should thus be willing to accept the first entailment in Greenberg's derivation and allow that RLI of an interacting $\tau$-function, appropriately defined, entails that the corresponding vacuum expectation value of an unordered product of fields satisfies WLC. ${ }^{25}$ However, a pragmatist is not in the position of accepting the second entailment in Greenberg's derivation, based as it is on Jost's purist proof. (Again, the technical difficulty is still the problem that interacting $\tau$-functions and their corresponding vacuum expectation values of unordered products of fields do not necessarily satisfy SC.) This does not preclude the possibility of a pragmatist version of this second entailment, however. It would require demonstrating that WLC of a vacuum expectation value of an unordered product of interacting fields (defined, perhaps, in terms of the Gell-Mann/Low formula) entails the existence of a CPT operator. Such a demonstration, however, has not appeared in the literature.

The upshot of this discussion is that Greenberg's demonstration fails to bridge the gap between pragmatists and purists. In the next section, I will consider what can be interpreted as another attempt at mediation, namely, causal perturbation theory.

\section{Causal Perturbation Theory}

The locus classicus of causal perturbation theory is (Epstein and Glaser [1973]). ${ }^{26}$ This approach consists of both a regularization scheme that explicitly addresses the purist's UV Problem, and an axiomatic scheme that can be interpreted as a purist attempt to address the pragmatist's Renormalization and Existence problems. These schemes, like the problems they address, can be separated. Indeed, the regularization scheme associated with causal perturbation theory can be adopted by pragmatist approaches to QFT, independently of the axiomatic scheme (Helling [unpublished]; Falk, Häußling, and Scheck [2010]). Conversely, the framework of causal perturbation theory has been incorporated into algebraic quantum field theory (AQFT) to produce a perturbative approach ('pAQFT') to this version of purism (see, e.g., Summers [2012], pp. 45-8, for a brief review). Finally, as part of their critique of Greenberg ([2002]), Dütsch and Gracia-Bondía ([2012]) prove a version of the CPT theorem within causal perturbation theory.

\footnotetext{
${ }^{25}$ More precisely, the proof of WLC still goes through if one replaces the Wightman functions $W^{(n)}$ in Greenberg's expression (7) with generic vacuum expectation values of unordered products of fields $\left\langle 0\left|\phi\left(x_{1}\right) \ldots \phi\left(x_{n}\right)\right| 0\right\rangle$.

${ }^{26}$ The formalism is thus sometimes referred to as the "Epstein-Glaser approach". Reviews can be found in (GraciaBondía [2006]; Pinter [2001]; Brunetti and Fredenhagen [2000]; Prange [1999]).
} 
These considerations suggest that causal perturbation theory offers a form of mediation in the debate between pragmatists and purists. The purpose of this section is to assess the status of this mediation.

In causal perturbation theory, the basic object is the $S$-matrix ( $a$ la Weinberg), but it is conceived as a formal power series in operator-valued distributions (à la Wightman). Epstein and Glaser ([1973]) base this approach on the following axioms:

(i) Formal $S$-matrix. The $S$-matrix is given by a formal power series in operator-valued distributions $S_{n}(g)$, where $g \in \mathcal{S}\left(\mathbb{R}^{4}\right)$ :

$$
S(g)=\sum_{n=0}^{\infty} \frac{-i^{n}}{n !} \int S_{n}\left(x_{1}, . ., x_{n}\right) g\left(x_{1}\right) \ldots g\left(x_{n}\right) d^{4} x_{1} \ldots d^{4} x_{n}
$$

Each term $S_{n}(g)=-i^{n} / n ! \int S_{n}\left(x_{1}, \ldots, x_{n}\right) g\left(x_{1}\right) \ldots g\left(x_{n}\right) d^{4} x_{1} \ldots d^{4} x_{n}$ in this sum is assumed to be an operator-valued distribution that corresponds to a linear operator on a dense subspace of a separable Hilbert space $\mathcal{H}$.

(ii)-(iv) The $S$-matrix satisfies Translation Invariance, Lorentz Invariance, and Unitarity.

(v) Causality. The $S$-matrix satisfies $S\left(g_{1}+g_{2}\right)=S\left(g_{2}\right) S\left(g_{1}\right)$, if the supports of $g_{1}$ and $g_{2}$ are spacelike separated.

Causality is essentially a distribution-theoretic version of Weinberg's Cluster Decomposition of Section 3.2. Esptein and Glaser were able to show that, given these axioms, the distributions $S_{n}$ in (8) can be generated inductively as time-ordered products of fields, provided that one sets $S_{0}=$ 1 (the identify on $\mathcal{H}$ ), and $S_{1}\left(x_{1}\right)=\mathscr{L}_{\text {int }}$, where $\mathscr{L}_{\text {int }}$ is the interaction Lagrangian density of a given RQFT. Moreover, the distributions so-generated are only defined in the complement of the origin; i.e., $S_{n} \in \mathcal{D}^{\prime}\left(\mathbb{R}^{4 n} \backslash\{0\}\right) .^{27}$ One should recall that Greenberg's purist UV Problem was that $n$-point $\tau$-functions (necessary for calculating the $S$-matrix) are also only defined in the complement of the origin. This suggests that, from a distribution-theoretic point of view, the purist $U V$ Problem is the problem of extending a distribution, defined in the complement of the origin, to one defined everywhere. A solution to this problem is briefly reviewed in the next section.

\subsection{A Purist Solution to the UV Problem}

The UV Problem for purists was how to define the product of the types of distributions that are of interest in interacting QFTs. The specific problem has now become that of extending a

\footnotetext{
${ }^{27}$ One can show that the Causality axiom entails the $S_{n}$ can be constructed as elements in $\mathcal{D}^{\prime}\left(\mathbb{R}^{4 n} \backslash D_{n}\right)$, where $D_{n}$ is the diagonal set of points in $\mathbb{R}^{4 n}$ (see Section 4.3). Translation invariance then allows this set to be identified with the origin. See, e.g., (Gracia-Bondía [2006], pp. 27-8; Prange [1999], p. 2232; Brunetti and Fredenhagen [2000], pp. 641-3).
} 
distribution defined on the complement of the origin to one defined everywhere. More precisely, given a distribution $t_{0} \in \mathcal{D}^{\prime}\left(\mathbb{R}^{n} \backslash\{0\}\right)$, the task is to construct an extension $t \in \mathcal{D}^{\prime}\left(\mathbb{R}^{n}\right)$, such that $t(f)=t_{0}(f), \forall f \in \mathcal{D}\left(\mathbb{R}^{n} \backslash\{0\}\right)$ (Prange [1999], p. 2226). One solution to this problem consists of the following two key ingredients. ${ }^{28}$

First, in order to have a sense of how a distribution behaves as the origin is approached, Steinmann ([1971]) defined the scaling degree of a distribution $t$ with respect to the origin as the greatest lower bound $\omega$ of the set $\left\{\omega^{\prime} \in \mathbb{R}: \lim _{\lambda \rightarrow 0} \lambda^{\omega^{\prime}} t(\lambda x)=0\right\}{ }^{29}$ One can now define the singular order $\rho$ of $t$ as the difference $\rho \equiv \omega-n$ between its scaling degree and the dimension of the configuration space. The singular order is a rigorous formulation, in the context of distributions, of the superficial degree of divergence associated with a Feynman diagram in pragmatist approaches to QFT (Gracia-Bondía [2006], p. 33). ${ }^{30}$

The second key ingredient consists of the following two results (Brunetti and Fredenhagen [2000], p. 645, 647):

Let $t_{0} \in \mathcal{D}^{\prime}\left(\mathbb{R}^{n} \backslash\{0\}\right)$ have scaling degree $\omega$ with respect to the origin. Then

(i) If $\omega<n$, there exists a unique extension $t \in \mathcal{D}^{\prime}\left(\mathbb{R}^{n}\right)$ of $t_{0}$ with scaling degree $\omega$.

(ii) If $\omega \geq n$, there exist extensions $t \in \mathcal{D}^{\prime}\left(\mathbb{R}^{n}\right)$ of $t_{0}$ with scaling degree $\omega$, and which are uniquely determined by their values on a finite set of test functions.

The second result is based on the fact that any function $f \in \mathcal{D}\left(\mathbb{R}^{n}\right)$ can be decomposed as $f=f_{1}+$ $f_{2}$, where (Brunetti and Fredenhagen [2000], p. 647):

$$
\begin{array}{ll}
f_{1}(x)=\sum_{|\alpha| \leq \rho}\left(\partial^{\alpha} f\right)(0) w_{\alpha}(x) & \text { for } w_{\alpha} \in \mathcal{D}\left(\mathbb{R}^{n}\right) \text { such that } \partial^{\alpha} w_{\beta}(0)=\delta_{\beta}^{\alpha}, \\
f_{2}(x)=\sum_{|\alpha|=|\rho|+1} x^{\alpha} \psi_{\alpha}(x) & \text { for } \psi_{\alpha} \in \mathcal{D}\left(\mathbb{R}^{n}\right) .
\end{array}
$$

The first term $f_{1}$ is a weighted version of the order $\rho$ Taylor polynomial of $f$ at $x=0,{ }^{31}$ and the second term $f_{2}$ is the remaining part of $f$ that vanishes up to order $\rho$ at the origin. If $\rho$ is identified as the singular order of $t_{0}$, then $t_{0}$ is well-defined on $f_{2}$, but not on $f_{1}$ (in particular, $t_{0}\left(w_{\alpha}\right)$ diverges). An extension $t$ of $t_{0}$ can be constructed by first setting $t\left(f_{2}\right)=t^{\prime}\left(f_{2}\right)$, where $t^{\prime}$ is

\footnotetext{
${ }^{28}$ The account below of the method of extending distributions is based on expositions found in (Keller [unpublished]; Brunetti and Fredenhagen [2000]; Prange [1999]). An alternative method involves 'splitting' distributions into advanced and retarded parts, and was developed by Epstein and Glaser ([1973]). For a recent exposition of this method, see (Aste et al. [2010]).

${ }^{29}$ See, e.g., (Brunetti and Fredenhagen [2000], p. 644; Prange [1999], p. 2226). The limit is meant in the sense of distributions.

${ }^{30}$ The superficial degree of divergence of a Feynman diagram is obtained by counting powers of momenta in the momentum space integral corresponding to the diagram.

${ }^{31}$ This can be made a bit more perspicuous by considering the example $w_{\alpha}(x)=\left(x^{\alpha} / \alpha !\right) w(x)$, with the weight function $w(x)=1$ on some neighborhood of the origin (Dütsch and Fredenhagen [2004], p. 1334).
} 
the unique extension of the distribution $x^{\alpha} t_{0}$ guaranteed by the first result (i) above, ${ }^{32}$ and then specifying the action of $t$ on $f_{1}$. The latter amounts to specifying the values $t\left(w_{\alpha}\right)=C_{\alpha} \in \mathbb{C}$. Thus the general form of an extension for result (ii) is given by (Dütsch and Fredenhagen [2004], p. 1310):

$$
t=t^{\prime}+\sum_{|\alpha| \leq \rho} C_{\alpha}\left(\partial^{\alpha} \delta\right)
$$

where the Dirac $\delta$-distribution is defined on test functions $f$ by $\left(\partial^{\alpha} \delta\right)(f) \equiv\left(\partial^{\alpha} f\right)(0)$. In words, (10) says that an extension $t$ of $t_{0}$ is obtained by adding a finite number of multiples of derivatives of the Dirac $\delta$-distribution to $t^{\prime}$. As Helling ([unpublished], p. 18) points out, the values of $C_{\alpha}{ }^{\prime}$... are exactly those where $\left[t_{0}\left(w_{\alpha}\right)\right]$ was undefined, and selecting a certain value corresponds [in pragmatist approaches] to picking a counter term..., as formally infinite values are replaced by finite ones...'. One can now use this method of extending distributions to obtain all the terms in (8) as uniquely defined distributions with corresponding Hilbert space operators. This amounts to a purist solution to the UV Problem.

\subsection{CPT Invariance}

Dütsch and Gracia-Bondía ([2012], pp. 430-1) have shown that if CPT invariance holds prior to regularization via the method of distribution extension, then it holds after regularization, too. In particular, they show that, under the assumptions that the free fields $\phi(x)$ in the time-ordered products $S_{n}\left(\phi\left(x_{1}\right) \ldots \phi\left(x_{n}\right)\right)$ in (8) are CPT invariant, and the interaction Lagrangian density $\mathscr{L}_{\text {int }}$ is CPT invariant, then the regularized time-ordered products of fields are CPT invariant. Schematically, Dütsch and Gracia-Bondía's CPT theorem thus takes the following form:

[(Causal perturbation theory axioms) \& (CPT invariance of free fields and interaction)] $\Rightarrow$ (CPT invariance of regularized time-ordered products of fields)

Thus CPT invariance 'survives' the process of distribution extension that solves the UV Problem. This is surely an advantage over the typical purist understanding of CPT invariance. It was argued in Section 3.1 above that, under the latter, CPT invariance can only be predicated of free (or trivially interacting) states. Dütsch and Gracia-Bondía demonstrate that CPT invariance is not limited to such states; rather, 'regularized' states can be said to possess it, too. Intuitively, such states are one step removed from free (or trivially interacting) states, and one step closer to the non-trivially interacting states of pragmatist's approaches.

One might now wonder how this compares with the pragmatist understanding of CPT invariance. In particular, it was argued in Section 3.2, that, under the latter, CPT invariance can be predicated of both free and interacting states (on pain of confronting the Renormalization Problem). Thus, given that CPT invariance can be said to hold of regularized states under the causal perturbation

\footnotetext{
${ }^{32}$ Since $f_{2} \in \mathcal{D}\left(\mathbb{R}^{d} \backslash\{0\}\right), t_{0}\left(f_{2}\right)=\sum_{|\alpha|=\rho \mid+1} x^{\alpha} t_{0}\left(\psi_{\alpha}\right)$ is well-defined, and one can show that the scaling degree of the distribution $x^{\alpha} t_{0}$ is less than $n$ (Brunetti and Fredenhagen [2000], p. 647); thus by result (i), $x^{\alpha} t_{0}$ has a unique extension, call it $t^{\prime}$.
} 
theorist's understanding, to what degree can it be said to hold of non-trivially interacting states? This is the subject of the next section.

\subsection{The Problem of Empirical Import and the Adiabatic Limit}

In the causal perturbation theory approach, there are two obstacles to interpreting the formal expression (8) as a non-trivial, interacting $S$-matrix. The first is that the formal test functions $g(x)$ that appear in it must be removed. These may be interpreted as a low-energy (IR) cut-off that regulates long-distance degrees of freedom in the interaction. As such, they address the IR problem for massless theories like QED (see footnote 15 above). Moreover, an IR cut-off also addresses the pragmatist's Problem (a) of Section 3.2 above (recall this generated the Renormalization Problem), as Brunetti and Fredenhagen observe:

Now in a translationally invariant theory, the interacting fields approach the asymptotic free fields only in a rather weak sense (LSZ-asymptotic condition [viz., (5)]). Moreover, the Haag Theorem forbids the construction of interacting fields in the vacuum Hilbert space of the time0 free fields. In the Epstein and Glaser scheme, these problems are, in a first step, circumvented by choosing interactions which take place only in a bounded region of spacetime. Then the scattering operator can be defined in the interaction picture as the time evolution operator from the past, before the interaction was switched on, to the future, after the interaction was switched off (Brunetti and Fredenhagen [2000], p. 626).

To remove the $g(x)$, one must take an 'adiabatic limit' $g(x) \rightarrow 1$. There are two versions of this limit: a weak version requires that the time-ordered products of fields generated from the $S$ matrix (8) exist as distributions in the limit $g(x) \rightarrow 1$, whereas a strong version requires, in addition, that the $S$-matrix itself (as a formal power series) exists as a unitary operator (see, e.g., Pinter [2001], p. 337). Note that the strong sense is a necessary (but not sufficient) condition for causal perturbation theory to address both the pragmatists' Existence Problem, and the purists' Problem of Empirical Import. Recall that the former is the problem of whether the perturbative power series expansion of the $S$-matrix actually exists as a convergent series. The latter is the problem of whether (non-trivial) interacting models of the causal perturbation theory axioms exist (and in particular, axiom 1). Thus, if the power series expansion (8) exists as a convergent series, or if there are (non-trivial) interacting models of axiom 1, then a corresponding unitary $S$ matrix operator must exist (this assumes that an appropriate notion of convergence for operators exists in the corresponding Hilbert space; i.e., that, if all the terms in (8) exist and converge to a well-defined expression (8), then likewise the operators corresponding to the terms in (8) converge to a well-defined operator). On the other hand, in a theory that only satisfies the weak adiabatic limit, only the individual terms in (8) are guaranteed to exist, as opposed to the full expansion itself. With this in mind, Pinter reports the following:

The weak adiabatic limit was proved to exist for massive theories, QED and massless $\lambda: \Phi^{2 n}$ : theories. The existence of the adiabatic limit in the strong sense has only been proved for massive theories (Pinter [2001], p. 337).

This suggests that only massive theories have a chance of being exactly formulated in causal perturbation theory. Whether or not they can be is just the second obstacle to interpreting (8) as 
a non-trivial interacting $S$-matrix mentioned at the start of this section. The question this second obstacle raises for the causal perturbation theorist is, Does the formal power series expression (8) for the $S$-matrix exist as a convergent series with a corresponding linear operator for non-trivial interacting RQFTs? This is simply the pragmatist's Existence Problem; namely, it is the problem of whether the perturbative power series expansion of the $S$-matrix (and/or relevant quantities of interest) exists as a convergent series for interacting RQFTs. In causal perturbation theory, since the existence of the $S$-matrix appears as one of the axioms, this pragmatist problem is simply the purist's Problem of Empirical Import; namely, it is the problem of whether a non-trivial interacting model of the causal perturbation theory axioms exists, in particular, axiom 1.

These observations indicate the sense in which causal perturbation theory, in seeking to provide a rigorous foundation for perturbation theory, can be viewed as mediating between pragmatism and purity. In particular, regardless of whether the adiabatic limit exists in either the weak or the strong sense, causal perturbation theory fails to address the purist's Problem of Empirical Import: there are no (non-trivial) interacting models of its axioms. On this account, causal perturbation theory is no different from other purist approaches. However, the fact that its axioms include a formal expression for the perturbative power series expansion of the $S$-matrix suggests that, in causal perturbation theory, the purist's Problem of Empirical Import is simply the pragmatist's Existence Problem. ${ }^{33}$

\section{Conclusion}

I'd like to conclude by first comparing casual perturbation theory with the standard bearers of pragmatism and purity, and then drawing two lessons and a general moral from this comparison. Wallace's ([2011]) cut-off QFT (CQFT), as a pragmatist approach, addresses the Renormalization Problem by expressing renormalized parameters as functions of a finite UV cut-off $\Lambda$, and then simply not taking $\Lambda$ to infinity. CQFT also solves the pragmatist's $U V$ Problem by employing $\Lambda$ to regulate divergent integrals in expressions like (2). A consequence of using such a permanent cut-off is that CQFT is not Lorentz invariant; but, as Wallace ([2011], p. 123) argues, one of the assumptions underlying CQFT is that it holds only for energy scales on the order of $\Lambda$, and conceivably Lorentz invariance could reappear at higher energy scales. Finally, CQFT leaves the pragmatist's Existence Problem unsolved. Fraser's ([2011]) axiomatic/algebraic QFT (AQFT), as a purist approach, and as typically formulated, leaves both the Problem of Empirical Import and the purist's UV Problem unsolved. Note, however, that there is nothing to stop an AQFTer from adopting the method of distribution extension as a solution to the latter. Moreover, perturbative AQFT (pAQFT) offers a means of incorporating the advantages of casual perturbation theory into the AQFT framework (see footnote 32). Finally, causal perturbation theory solves the

\footnotetext{
${ }^{33}$ The application of causal perturbation theory to AQFT (i.e., "pAQFT") is associated with an 'algebraic adiabatic limit'. Whereas the weak and strong adiabatic limits are characterized by holding fields and states fixed in the asymptotic past and future, the algebraic adiabatic limit is characterized by holding the fields fixed in regions in the interior of spacetime (Holland and Wald [2003], p. 136). Summer ([unpublished], pp. 47-8) indicates that, while pAQFT 'falls comfortably within the framework of AQFT', it faces what this essay has referred to as the Existence Problem and the Problem of Empirical Import: '[T]aking expectations of observables in states in this approach results in a formal complex power series, not a complex number. Hence, in order to make the connection to experiments one must deliberately consider a partial sum of this series, i.e. consider the perturbation series only to a finite order, as is done in heuristic QFT. Since these series are not convergent, one is returned to the question, "Is there an exact model?".'
} 
purist's $U V$ problem (for instance, by the method of distribution extension); and while it cannot be said to solve the purist's Problem of Empirical Import, it can be interpreted as converting this purist problem into the pragmatist's Existence Problem.

This comparison provides two lessons for the debate between pragmatists and purists. First, problems associated with renormalization should be made distinct from problems associated with perturbation theory. In particular, the pragmatist's Renormalization Problem is conceptually distinct from the pragmatist's UV Problem and Existence Problem, the latter being problems associated (indirectly and directly) with perturbation theory. This suggests that purists need not completely reject the tools of renormalized perturbation theory that underlie the empirical success of pragmatist approaches: One can object to renormalization as mathematically suspect while engaging rigorously with perturbation theory. Second, the purist Problem of Empirical Import is not necessarily conceptually distinct from the pragmatist Existence Problem. This suggests that pragmatists should acknowledge that purists may be taken to task on the Problem of Empirical Import to the same extent that pragmatists may be taken to task on the Existence Problem.

The above discussion also suggests a general moral for the debate between pragmatists and purists. As we've seen, both approaches face particular sets of problems. In the absence of a formulation of RQFTs that is entirely problem-free, it would seem that the best approach to foundational issues would be to employ all available formulations, being aware of the types of problems any given formulation faces. Thus, for instance, in addressing the question, 'Why is CPT invariance an essential property of RQFTs?', it helps to consider both pragmatist and purist formulations of the CPT theorem, as opposed to focusing on any one particular formulation. Moreover, as Section 5 demonstrated, an appreciation of the problems associated with alternative formulations of the CPT theorem can help dispel potential misunderstandings associated with it. In general, the existence of alternative formulations of a theory should not be something to despair over; rather it should be embraced. Such alternatives indicate a robust and fertile theory with sufficiently complex core structure to underwrite multiple non-trivial realizations. 


\section{Appendix}

Greenberg's ([2002]) proof that, at a Jost point, RLI of $\tau$-functions entails WLC of Wightman functions may be demonstrated as follows (after Dütsch and Gracia-Bondía [2012], pp. 432-3). We wish to show that, at a Jost point, any Wightman function satisfies $W\left(x_{1}, \ldots, x_{n}\right)=$ $W\left(x_{1}, \ldots, x_{n}\right)$. We first assume Wightman functions satisfy RLI; that is, we assume there is a representation of the restricted Lorentz group, $L_{+}^{\uparrow} \ni \Lambda \mapsto U(\Lambda)$, such that

$$
W\left(\Lambda x_{1}, \ldots \Lambda x_{n}\right)=U(\Lambda) W\left(x_{1}, \ldots, x_{n}\right) U(\Lambda)^{-1}, \quad \forall \Lambda \in L_{+}^{\uparrow}
$$

RLI of $\tau$-functions is the analogous assumption that

$$
\tau\left(\Lambda x_{1}, \ldots \Lambda x_{n}\right)=U(\Lambda) \tau\left(x_{1}, \ldots, x_{n}\right) U(\Lambda)^{-1}, \quad \forall \Lambda \in L_{+}^{\uparrow}
$$

Now suppose $\left(x_{1}, \ldots, x_{n}\right)$ is a Jost point (see footnote 4$)$. This means that $\left(x_{i}-x_{j}\right)$ is spacelike for all $x_{i}, x_{j} \in\left(x_{1}, \ldots, x_{n}\right), i \neq j$. For such points, it is always possible to choose two Lorentz transformations $\Lambda_{\infty}, \Lambda_{\beta} \in L_{+}^{\uparrow}$ such that they time-order $\left(x_{1}, \ldots, x_{n}\right)$ in opposite ways; i.e., $\left(\Lambda_{\alpha} x_{1}\right)^{0}>$ $\ldots>\left(\Lambda_{1} x_{n}\right)^{0}$ and $\left(\Lambda_{\beta} x_{1}\right)^{0}<\ldots<\left(\Lambda_{\beta} x_{n}\right)^{0}$. Then

$$
\begin{aligned}
W\left(x_{1}, \ldots, x_{n}\right) & =U\left(\Lambda_{\alpha}\right)^{-1} W\left(\Lambda_{\alpha} x_{1}, \ldots \Lambda_{\alpha} x_{n}\right) U\left(\Lambda_{\alpha}\right) & & \text { (A1) } \\
& =U\left(\Lambda_{\alpha}\right)^{-1} \tau\left(\Lambda_{\alpha} x_{1}, \ldots \Lambda_{\alpha} x_{n}\right) U\left(\Lambda_{\alpha}\right) & & \text { Definition of } \Lambda_{\alpha} \text { and (7) } \\
& =\tau\left(x_{1}, \ldots, x_{n}\right) & & \text { (A2) } \\
& =U\left(\Lambda_{\beta}\right)^{-1} \tau\left(\Lambda_{\beta} x_{1}, \ldots \Lambda_{\beta} x_{n}\right) U\left(\Lambda_{\beta}\right) & & \text { (A2) } \\
& =U\left(\Lambda_{\beta}\right)^{-1} W\left(\Lambda_{\beta} x_{n}, \ldots \Lambda_{\beta} x_{1}\right) U\left(\Lambda_{\beta}\right) & & \text { Definition of } \Lambda_{\beta} \text { and (7) } \\
& =W\left(x_{n}, \ldots, x_{1}\right) & & \text { (A1) }
\end{aligned}
$$




\section{References}

Araki, H. [1999]: Mathematical Theory of Quantum Fields, Oxford: Oxford University Press.

Arntzenius, F. [2011]: 'The CPT Theorem', in C. Callender (ed.), 2011, The Oxford Handbook of Philosophy of Time, Oxford: Oxford University Press, pp. 633-46.

Arntzenius, F. and Greaves, H. [2009]: 'Time Reversal in Classical Electromagnetism', British Journal for the Philosophy of Science, 60, pp. 557-84.

Aste, A., von Arx, C. and Scharf, G. [2010]: 'Regularization in Quantum Field Theory from the Causal Point of View', Progress in Particle and Nuclear Physics, 64, pp. 61-119.

Bain, J. [2011]: 'CPT Invariance, the Spin-Statistics Connection, and the Ontology of Relativistic Quantum Field Theories', Erkenntnis. DOI 10.1007/s10670-011-9324-9.

Bain, J. [2000]: 'Against Particle/Field Duality: Asymptotic Particle States and Interpolating Fields in Interacting QFT (Or: Who's Afraid of Haag's Theorem?)', Erkenntnis, 53, pp. 375-406.

Bouatta, N. and Butterfield, J. [unpublished]: 'On Emergence in Gauge Theories at the 't Hooft Limit', <philsci-archive.pitt.edu/id/eprint/9288>.

Brunetti, R. and Fredenhagen, K. [2000]: 'Microlocal Analysis and Interacting Quantum Field Theories: Renormalization on Physical Backgrounds', Communications in Mathematical Physics, 208, pp. 623-61.

Duncan, A. [2012]: The Conceptual Framework of Quantum Field Theory, Oxford: Oxford University Press.

Dütsch, M. and Fredenhagen, K. [2004]: 'Causal Perturbation Theory in Terms of Retarded Products, and a Proof of the Action Ward Identity', Reviews in Mathematical Physics, 16, pp. 1291-348.

Dütsch, M. and Gracia-Bondía, J. [2012]: 'On the Assertion that PCT Violation Implies Lorentz Non-invariance', Physics Letters, B711, pp. 428-33.

Earman, J. and Fraser, D. [2006]: 'Haag's Theorem and its Implications for the Foundations of Quantum Field Theory', Erkenntnis, 64, pp. 305-44.

Epstein, H. and Glaser, V. [1973]: 'The Role of Locality in Perturbation Theory', Annales de l'Institut Henri Poincaré, A19, pp. 211-95.

Falk, S., Häußling, R. and Scheck, F. [2010]: 'Renormalization in Quantum Field Theory: An Improved Rigorous Method', Journal of Physics, A43, pp. 035401(1)-(15).

Fraser, D. [2011]: 'How to Take Particle Physics Seriously: A Further Defense of Axiomatic Quantum Field Theory', Studies in History and Philosophy of Modern Physics, 42, pp. 126-35. 
Fraser, D. [2009]: Quantum Field Theory: Underdetermination, Inconsistency, and Idealization', Philosophy of Science, 76, pp. 536-67.

Gell-Mann, M. and Low, F. [1951]: 'Bound States in Quantum Field Theory', Physical Reivew, 84, pp. 350-4.

Gracia-Bondía, J. [2006]: 'The Epstein-Glaser Approach to QFT', AIP Conference Proceedings, 809, pp. 24-43.

Greaves, H. [2010]: 'Towards a Geometrical Understanding of the CPT Theorem', British Journal for the Philosophy of Science, 61, pp. 27-50.

Greaves, H. and T. Thomas [unplublished]: 'The CPT Theorem', <arXiv:1204.4674v1>.

Greenberg, O. [2006]: 'Why is CPT Fundamental?', Foundations of Physics, 36, pp. 1535-53.

Greenberg, O. [2002]: 'CPT Violation Implies Violation of Lorentz Invariance', Physical Review Letters, 89, pp. 231602(1)-(4).

Haag, R. [1996]: Local Quantum Physics, Berlin: Springer Verlag.

Hagar, A. [2009]: 'Minimal Length in Quantum Gravity and the Fate of Lorentz Invariance', Studies in History and Philosophy of Modern Physics, 40, pp. 259-67.

Halvorson, H. and Müger, M. [2007]: 'Algebraic Quantum Field Theory', in J. Butterfield and J. Earman (eds), 2007, Philosophy of Physics, Elsevier, pp. 731-922.

Helling, R. [unpublished]: 'How I Learned to Stop Worrying and Love QFT', $<$ arxiv.org/abs/1201.2714>.

Hollands, S. and Wald, R. [2003]: 'On the Renormalization Group in Curved Spacetime', Communications in Mathematical Physics, 237, pp. 123-60.

Jost, R. [1957]: 'Eine Bemerkung zum CTP Theorem', Helvetica Physica Acta, 30, pp. 409-16.

Kaku, M. [1993]: Quantum Field Theory, Oxford: Oxford University Press.

Kanwal, R. [2004]: Generalized Functions: Theory and Applications, 3rd Edition, Berlin: Springer Verlag.

Keller, K. [unpublished]: 'Dimensional Regularization in Position Space and a Forest Formula for Regularized Epstein-Glaser Renormalization', <http://arxiv.org/abs/1006.2148>.

Kostelecky, V. (ed.) [2011]: Proceedings of the 5th Meeting on CPT and Lorentz Symmetry, World Scientific. 
Lehmann, H., Symanzik, K., and Zimmermann, W. [1955]: 'Zur Formulierung quantisierter Feldtheorien', Nuovo Cimento, 1, pp. 205-25.

Maggiore, M. [2005]: A Modern Introduction to Quantum Field Theory, Oxford: Oxford University Press.

Pinter, G. [2001]: 'Finite Renormalizations in the Epstein Glaser Framework and Renormalization of the $S$-matrix of $\Phi^{4}$-Theory', Annalen der Physik, 10, pp. 333-63.

Prange, D. [1999]: 'Epstein-Glaser Renormalization and Differential Renormalization', Journal of Physics, A32, pp. 2225-38.

Reed, M. and Simon, B. [1975]: Methods of Modern Mathematical Physics, Vol. II: Fourier Analysis, Self-Adjointness, New York: Academic Press.

Ryder, L. [1996]: Quantum Field Theory, Cambridge: Cambridge University Press.

Srednicki, M. [2007]: Quantum Field Theory, Cambridge: Cambridge University Press.

Steinmann, O. [2000]: Perturbative Quantum Electrodynamics and Axiomatic Field Theory, Berlin: Springer Verlag.

Steinmann, O. [1971]: Perturbation Expansions in Axiomatic Field Theory, Berlin: Springer Verlag.

Streater, R. and Wightman, A. [2000, (1964)]: PCT, Spin and Statistics, and All That, Princeton: Princeton University Press.

Summers, S. [unpublished]: 'A Perspective on Constructive Quantum Field Theory', $<$ http://arxiv.org/abs/1203.3991>.

Wallace, D. [2011]: 'Taking Particle Physics Seriously: A Critique of the Algebraic Approach to Quantum Field Theory', Studies in History and Philosophy of Modern Physics, 42, pp. 116-25.

Weinberg, S. [1995]: The Quantum Theory of Fields, Vol. 1, Cambridge University Press.

Weinberg, S. [1964]: 'Feynman Rules For Any Spin', Physical Review, 133, pp. B1318-32. 